\title{
IN DEPTH STUDY OF EPIDEMIOLOGY IN TRAUMATIC SPINAL CORD INJURY PATIENTS PRESENTED TO THE MAIN SPINAL REHABILITATION UNIT IN SRI LANKA
}

\author{
Appuhamy H.S.D ${ }^{1}$, Munidasa S.M.P.D ${ }^{2}$, Suriarachchi J.N ${ }^{3}$, Sirigampala S.A.N ${ }^{4}$ and \\ Ratnayake V.S.K ${ }^{5}$ \\ 1, 2,3,4,5 Rheumatology and Medical Rehabilitation, Rheumatology and Rehabilitation Hospital, Ragama, Sri \\ Lanka
}

\begin{abstract}
Spinal cord injury (SCI) has high mortality and morbidity. Prevention is the best way to reduce the burden on health sector and society. Lack of a large scale referral study on epidemiology of SCI in Sri Lanka, is a barrier to improve methods of prevention. A descriptive (prospective) study was conducted in Rheumatology and Rehabilitation Hospital, Ragama, where randomly selected 42 patients diagnosed of traumatic spinal injuries were presented with an interviewer-administered questionnaire after consent. Mean age of the study population was 32.74 years (SD 11.94), where $86.36 \%$ of them were males. $61.9 \%$ were married. $85.7 \%$ were occupied, out of which the majority had engaged in elementary occupations $(33.34 \%)$. Only $28.6 \%$ had secondary or higher education. Most of the injuries (44.4\%) were due to falls occurred during occupational activities. Other significant etiologies contributed include falls during recreational / house hold activities and road traffic accidents with $22.7 \%$ and $22.2 \%$ respectively. Interestingly $78.94 \%$ of the injuries occurred during weekdays while $47.37 \%$ of the injuries took place during routine working hours ( 8.00 am to $4.00 \mathrm{pm})$. The majority had thoracic spinal injuries (55.56\%), while $33.2 \%$ and $11.2 \%$ had cervical and lumbosacral injuries respectively. 52.4\% had severe presentation with complete ASIA (American Spinal Injury Association) level "A" injuries. The final analysis indicates that traumatic SCI are more common among young males with low level of education, who engaged in elementary occupations. Hence these populations should be mainly targeted when planning methods of SCI prevention in Sri Lanka.
\end{abstract}

Keywords: Spinal, injury, epidemiology

\section{INTRODUCTION}

Spinal cord injury (SCI) is an insult to the spinal cord resulting in a change, either temporary or permanent, in the cord's normal motor, sensory, or autonomic function. Patients with spinal cord injuries usually have permanent and often devastating neurological deficits and disability. It was labeled as "an ailment not to be treated" in the Edwin Smith papyrus 5000 years ago. Unfortunately not much has changed, in many parts of the world known as the underdeveloped countries.

The incidence and prevalence of spinal injuries have been increasing, with the incidence rate estimated at

Corresponding Author Email:savidyadhanushka@gmail.com
15 to 40 cases per million worldwide, although injury prevention initiatives have tried to reduce the occurrence of SCIs (1). Spinal cord injuries can be divided into traumatic and non-traumatic injuries. The four main causes of injury were traumatic injuries, including traffic accidents, being struck by falling objects, crushing injuries and high falls, followed by low falls, violence, non-traumatic causes, sports-related injuries, and other unknown causes (2).

Spinal cord injuries are highly disabling and concentrated in young adults. As shown by the data of other studies, SCIs had affected with a male to female ratio of 2.5:1 and the average age at injury was $35.5+/-15.1$ years $(35.4+/-14.8$ for males and 
35.9+/-16.0 for females), which cause substantial burden for the affected individuals, their families and society. (3). But unfortunately in Sri Lanka there are no large scale studies regarding epidemiology of spinal cord injuries so far.

\section{METHODS}

The study was designed as a prospective descriptive study which was conducted at the Rheumatology and Rehabilitation Hospital (RRH) Ragama, which has 270 beds dedicated to Rheumatology and Rehabilitation, of which 125 beds are exclusive for spinal cord injury patients.

Patients with traumatic spinal injuries who admitted to RRH during this study period were included \& the patients with non traumatic spinal injuries were excluded. All the patients satisfying the inclusion criteria were recruited and data collection was proceed until the minimum sample size was achieved.

Interviewer administered questionnaire and a data extraction tool was used after pretesting. Face validity and consensual validity were assured beforehand. The investigators collected data by themselves from the patients or guardians.

\section{RESULTS}

Mean age of the study population was 32.74 years (SD 11.94), where $86.36 \%$ of them were males. $61.9 \%$ were married. $85.7 \%$ were occupied, out of which the majority had engaged in elementary occupations $(33.34 \%)$. SCI were more common among people with low level of education as only $28.6 \%$ had secondary or higher education level. The majority $(76.19 \%)$ were referred to us from the National Hospital of Sri Lanka.

Most of the injuries (44.4\%) were due to falls occurred during occupational activities. Other significant etiologies contributed include falls during recreational / house hold activities and road traffic accidents with occurrences of $22.7 \%$ and $22.2 \%$ respectively. Interestingly $78.94 \%$ of the injuries occurred during weekdays, while $47.37 \%$ of the injuries took place during routine working hours, which was defined as from 8.00 am to $4.00 \mathrm{pm}$.
In $94.4 \%$ SCI were due to blunt injuries while remaining had penetrating trauma. 95.24\% had vertebral injuries and $28.57 \%$ had associated other injuries when admitting to our hospital. $26.32 \%$ had multiple injuries and $50 \%$ of the population had disc or posterior ligamentous complex injures. The majority $(55.56 \%)$ had level 2 spinal cord injuries according to the ICD-1O classification. The majority had thoracic (T1-T12) spinal injuries (55.56\%), while $33.2 \%$ and $11.2 \%$ had cervical and lumbosacral spinal injuries respectively. 52.4\% had complete ASIA (American Spinal Injury Association) level A injuries while $47.6 \%$ had incomplete injuries out of which $9.5 \%$ and $38.1 \%$ had ASIA B \& C level injuries respectively.

The average time of delay to the Rheumatology and Rehabilitation Hospital (RRH) was 126.05 days, which ranged from 15 to 703 days. Mean length of hospital stay for rehabilitation in our hospital was 143.5 days (SD 69.28).

\section{DISCUSSION}

Most of the socio-demographic data of our study are compatible with other international studies. Majority of the population affected were married young males with low level of education, who were engaged in elementary, agricultural or fishery occupations, which indicate the effect of traumatic spinal injuries to the economy of the country as most were an important part of the nations' work force.

Risk factor analysis revealed that $94.4 \%$ SCI were blunt injuries where the majorities were due to falls occurred during occupational activities, followed by road traffic accidents and falls during recreational / house hold activities respectively. $78.9 \%$ of the injuries occurred during weekdays while $47.3 \%$ of the injuries took place during routine working hours. We believe that these data can be extremely useful in selecting target populations for future SCI prevention programmes in the region.

Injury severity analysis revealed that majority had thoracic spinal injuries with $95.24 \%$ had vertebral injuries and $50 \%$ of the population had disc or posterior ligamentous complex injures.

52.4\% had severe complete ASIA (American Spinal Injury Association) level $A$ injuries. However, we 
firmly believe that data on disease severity has been biased by the fact that as a well established tertiary care center most of the patients referred to us have severe injuries with significant disabilities.

A significant delay in admitting these patients to the RRH for long term rehabilitation was noted indicating that urgent actions are warranted in improving the knowledge on importance of rehabilitation among SCI patients and health care workers. It also indicates that further studies are needed to analyze the factors which have contributed to this significant delay.

\section{CONCLUSION}

Prevention is the best way to reduce the burden of SCI on health sector and society. SCI epidemiology and risk factors identified in this study can be highly useful in improving methods of prevention.

\section{REFERENCES}

Jackson AB, Dijkers M, Devivo MJ, Poczatek RB (2004) A demographic profile of new traumatic spinal cord injurychanges and stability over 30 years. Arch Phys Med Rehabil 85: 1740-1748. doi: 10.1016/j.apmr.2004.04.035

Chun-xia H, Jian-jun L, Hong-jun Z (2007) Epidemiology Characteristics of Spinal Cord Injury in Hospital: 1264 Cases Report. Chinese journal of Rehabilitation Theory and Practice 13: 1011-1013.

Karacan I, Koyuncu H, Pekel O, Traumatic spinal cord injuries in Turkey: a nation-wide epidemiological study. Spinal Cord 2000 Nov; 38(11):697-701.

http://www.health.gov.lk

Personal communicates from relevant hospitals 